\title{
Effects of Shaddock (Citrus maxima) Fruit Juice on Glucose Tolerance and Lipid Profile in Type-II Diabetic Rats
}

\author{
A. OYEDEPOT ${ }^{*}$ and S.O. BABARINDE
}

Department of Biological Sciences, Adeleke University, Ede P.M.B. 250, Ede, Osun State, Nigeria

oyedepo004tope@yahoo.com

Received 8 June 2012 / Accepted 22 June 2012

\begin{abstract}
Cirus maxima commonly known as Shaddock has been proved for hypoglycaemic effects. The objective of the present study was to evaluate the effects of Shaddock (C.maxima) fruit juice on glucose tolerance and lipid profile in streptozotocin (STZ) induced type-II diabetic rat. Two days old neonatal pups (7-10 g) were used and they were made diabetic by intraperitoneally (i.p.) injection of $90 \mathrm{mg} / \mathrm{kg} \mathrm{STZ}$ in citrate buffer solution. Different groups of animals were treated by 25\% and 50\% Shaddock fruit juice (SFJ) for 8 weeks. After 8 weeks treatment biochemical parameters from blood serum were analyzed. The significant differences of glucose, cholesterol, HDL, LDL, triglyceride, in $50 \%$ SFJ treated group compare to diabetic group were found. Therefore we conclude from this present study that Shaddock fruit juice has beneficial effects on glucose tolerance and lipid profile in streptozotocin-induced type-II diabetic rat.
\end{abstract}

Keywords: Shaddock fruit juice (SFJ), Diabetes mellitus, Blood Glucose, Cholesterol, Lipid parameters

\section{Introduction}

Diabetes mellitus is a disorder that affects the body's ability to make or use insulin. Insulin is a hormone produced in the pancreas that helps transport glucose (blood sugar) from the blood stream into the cells so they can break it down and use it for fuel. People cannot live without insulin ${ }^{1}$. Diabetes results in abnormal levels of glucose in the blood stream. This can cause severe short-term and longterm consequences ranging from brain damage to amputations and heart disease ${ }^{1}$.

Despite considerable progress in the treatment of diabetes by oral hypoglycemic agents, search for newer drugs continues because the existing synthetic drugs have several limitations. Pharmacotherapy of diabetes without any side effects is still a challenge ${ }^{2}$. So there is the need for complementary and alternative medicine with anti-diabetic activity and less side effects. Alternative therapies with anti-diabetic activity have been researched extensively. Ideal therapies should have a similar degree of efficacy without the troublesome side effects associated with conventional treatments ${ }^{3}$. Alternative treatments for diabetes have become increasingly popular in the last several years, including medicinal herbs, nutritional supplementation, acupuncture and hot tub therapy ${ }^{4}$. 
Hyperglycemia is involved in the etiology of development of diabetic complications. Hypoglycemic herbs increase insulin secretion, enhance glucose uptake by adipose or muscle tissues and inhibit glucose absorption from intestine and glucose production from liver $^{5}$.

Citrus fruits have been recommended in traditional herbal medicine as the source of diabetic medication or remedy for diabetes ${ }^{6}$. The citrus fruits are well recognized for their various ethno medicinal uses. These properties are attributed to their flavonoids and limonoids which are proven to possess anti-inflammatory and antitumor effects ${ }^{7}$. The peels are rich in pectin which is known to possess blood sugar lowering and cholesterol lowering properties $^{8}$. Sriparna et al., ${ }^{9}$ demonstrated the antihyperglycemic effect and antioxidant property of C. maxima leaf in streptozotocin induced diabetic rats. Similarly, a previous study ${ }^{10}$ has shown that the fruit juice of Shaddock (C.maxima) possess hypoglycemic and hypocholesterolemic activities.

In view of the various effects of $C$. maxima juice, this study was designed to assess the effect of Shaddock fruit juice on glucose tolerance and lipid profile in streptozotocininduced type -II diabetic rats.

\section{Experimental}

Neonatal rats $(7-10 \mathrm{~g})$ were used in this experimental. The experimental protocol was carried out as approved by Institutional Animal Ethics Committee and animals were maintained under standard conditions in an animal house.

\section{Preparation of bitter gourd fruit juice}

Shaddock fruits (SF) were obtained from the local market, washed thoroughly and fresh juice was prepared on a juicer eliminating seeds. It was centrifuged at $1000 \mathrm{rpm}$ on a tabletop refrigerated centrifuge for $10 \mathrm{~min}$ at $4{ }^{\circ} \mathrm{C}$. The clear supernatant was considered as $100 \%$ Shaddock fruit juice (SFJ) which was diluted with autoclaved distilled water to make 25 and $50 \%$ juice or any other desired concentrations ${ }^{11}$. The $100 \%$ juice was stored at $4{ }^{\circ} \mathrm{C}$.

\section{Induction of diabetes mellitus}

Wistar rats from an inbred colony were bred under well controlled conditions of temperature $\left(22 \pm 3^{\circ} \mathrm{C}\right)$, humidity $(55 \pm 5 \%)$ and $12 \mathrm{~h} / 12 \mathrm{~h}$ light-dark cycle. Conventional laboratory diet and tap water were provided ad libitum. Two days old neonates of the Wistar rats were injected intraperitoneally (i.p.) with $90 \mathrm{mg} / \mathrm{kg} \mathrm{STZ}$ in citrate buffer solution. Control neonates received equivalent amount of isotonic saline alone. The neonates (n2 STZ rats) were left with their own mothers and weaned at four weeks of age. Twelve weeks after the injection of STZ, animals were checked for fasting glucose levels.

\section{Treatment protocol}

Animals showing hyperglycemia (>140 mg/dL serum glucose level), 12 weeks after the injection of STZ were selected for the experiment as diabetics. Control animals injected with $0.1 \mathrm{~mol} / \mathrm{L}$ citrate buffer were used as normal control. Control rats were randomly divided into 3 groups; similarly, diabetic rats were divided in 3 groups. The experimental animals were divided into 6 groups, six animals in each group.

Group-1: Normal control (CON)

Group-2: Normal treated with 25\% SFJ $(10 \mathrm{~mL} / \mathrm{kg})\left(\mathrm{CSFJ}_{25}\right)$

Group-3: Normal treated with 50\% SFJ (10 mL/kg) (CSFJ 50 
Group-4: Diabetic control (DC)

Group-5: Diabetic treated with 25\% SFJ $(10 \mathrm{~mL} / \mathrm{kg})\left(\mathrm{DSFJ}_{25}\right)$

Group-6: Diabetic treated with 50\% SFJ (10 mL/kg) (DSFJ $\left.{ }_{50}\right)$

All the studies were carried for a period of eight weeks. During the study food intake, water intake and body weight of animals were recorded in all the groups. At the end of the study period, blood samples were collected under fasting conditions. For glucose tolerance test the animals were orally administered with $1.5 \mathrm{~g} / \mathrm{kg}$ of glucose and blood samples were collected after 30,60 and 120 minutes of oral glucose administration. Serum was separated by centrifugation at $5000 \mathrm{rpm}$ for $20 \mathrm{~min}$ and stored at $-20^{\circ} \mathrm{C}$ until the analysis was carried out. Serum samples were analyzed for glucose, cholesterol, HDLcholesterol, triglyceride using diagnostic kits (Span Diagnostics Ltd., India) and colorimetrically using UV Visible spectrophotometer (Shimadzu, Japan). VLDL and LDL were calculated as per Friedewald'sequation ${ }^{12}$.

\section{Statistical analysis}

Results are presented as Mean + SEM. Statistical differences between the means of the various groups were evaluated using one-way analysis of variance (ANOVA) followed by Tuckey's test. Data were considered statistically significant at $\mathrm{P}$ value $<0.05$.

\section{Results and Discussion}

\section{General features of experimental rats}

Intravenous injection of $90 \mathrm{mg} / \mathrm{kg}$ STZ in 2 day old rats produced signs of type-II diabetes which include loss of body weight, polyphagia and polydipsia after 12 weeks. Treatment with 50\% SFJ possibly reduced the elevated food and water intake of diabetic rats (Table 1).

Table 1. Effect of SFJ treatment on general features of control and diabetic rats

\begin{tabular}{ccccccc}
\hline Parameter & $\mathrm{CN}$ & $\mathrm{CSFJ}_{25}$ & $\mathrm{CSFJ}_{50}$ & $\mathrm{DC}$ & $\mathrm{DSFJ}_{25}$ & $\mathrm{DSFJ}_{50}$ \\
\hline $\begin{array}{c}\text { Body weight } \\
\text { after treatment, g }\end{array}$ & $251.32 \pm 10.22$ & $251.01 \pm 4.15$ & $250.43 \pm 11.43$ & $190.34 \pm 23.2^{*}$ & $204.2 \pm 4.56$ & $239.34 \pm 16.46$ \\
$\begin{array}{c}\text { Food intake } \\
\text { (g/animal/day) }\end{array}$ & $17.19 \pm 2.03$ & $16.50 \pm 2.49$ & $18.12 \pm 1.23$ & $22.38 \pm 1.11^{*}$ & $21.67 \pm 0.22$ & $20.78 \pm 0.56^{\#}$ \\
$\begin{array}{c}\text { Water intake } \\
\text { (mL/animal/day) }\end{array}$ & $27.34 \pm 0.71$ & $28.22 \pm 0.34$ & $27.21 \pm 0.32$ & $33.89 \pm 0.34^{*}$ & $30.78 \pm 0.66$ & $31.72 \pm 0.29^{\#}$ \\
\hline
\end{tabular}

$C N=$ Normal control; CSFJ $25=$ Normal treated with $25 \%$ SFJ; CSFJ 50=Normal treated with $50 \%$ SFJ; DC = Diabetic control; DSFJ 25=Diabetic treated with 25\% SFJ; DSFJ 50= Diabetic treated with 50\% SFJ. Values are expressed as Mean + S.E.M, * - significantly different from control $(p<0.05)$, \# - significantly different from diabetic control $(p<0.05)$

\section{Biochemical parameters}

In this study, STZ-diabetic rats were found to exhibit significant $(\mathrm{P}<0.05)$ hyperglycemia compared to control rats in oral glucose tolerance which was improved by $50 \%$ SFJ treatment (Table 2 and Figure 1). The study also recorded significant $(\mathrm{P}<0.05)$ increase in cholesterol, very low density lipoprotein (VLDL) and triglycerides levels, while there was significant $(\mathrm{P}<0.05)$ decrease in high density lipoprotein $(\mathrm{HDL})$-cholesterol levels in STZ diabetic rats as compared to control rats. Treatment with $50 \%$ SFJ significantly $(\mathrm{P}<0.05)$ reduced the cholesterol, VLDL and triglyceride levels in diabetic rats and increased the HDL-cholesterol levels. 25\% SFJ showed no significant effects on glucose level and lipid profile (Table 3 and Figure 2). 
Table 2. Effect of SFJ treatment on oral glucose tolerance test of control and diabetic rats

\begin{tabular}{ccccccc}
\hline $\begin{array}{c}\text { Serum glucose } \\
(\mathrm{mg} / \mathrm{dL}) \text { at } \\
\text { time interval }\end{array}$ & $\mathrm{CN}$ & $\mathrm{CSFJ}_{25}$ & $\mathrm{CSFJ}_{50}$ & $\mathrm{DC}$ & $\mathrm{DSFJ}_{25}$ & DSFJ $_{50}$ \\
\hline $30 \mathrm{~min}$ & $124.56 \pm 0.35$ & $128.45 \pm 0.93$ & $134.88 \pm 1.22$ & $255.11 \pm 11.34^{*}$ & $229.80 \pm 1.34$ & $169.79 \pm 0.33^{\#}$ \\
$60 \mathrm{~min}$ & $115.67 \pm 3.45$ & $119.78 \pm 2.45$ & $124.52 \pm 0.98$ & $241.69 \pm 3.01^{*}$ & $217.93 \pm 1.55$ & $144.40 \pm 0.63^{\#}$ \\
$120 \mathrm{~min}$ & $106.98 \pm 1.30$ & $108.46 \pm 0.91$ & $114.99 \pm 0.87$ & $231.33 \pm 2.05^{*}$ & $201.10 \pm 1.88$ & $124.52 \pm 3.05^{\#}$ \\
\hline
\end{tabular}

$C N=$ Normal control; CSFJ 25 = Normal treated with 25\% SFJ; CSFJ 50=Normal treated with 50\% SFJ; DC = Diabetic control; DSFJ 25=Diabetic treated with 25\% SFJ; DSFJ 50= Diabetic treated with 50\% SFJ. Values are expressed as Mean + S.E.M, * - significantly different from control $(p<0.05)$, \# - significantly different from diabetic control $(p<0.05)$

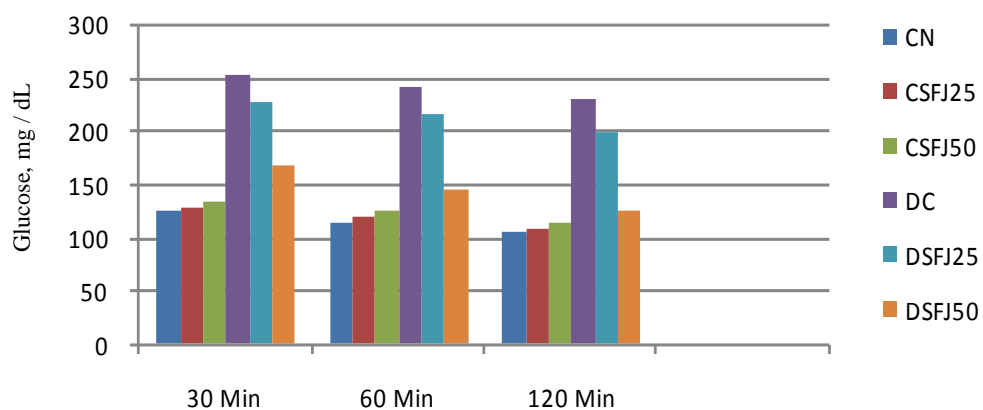

All values are means $\pm S . E(n=6) .{ }^{*} P<0.05$ compared with the native control group that reveals significant difference between two groups; \# $P<0.05$ compared with the diabetic group that reveals significant difference between two groups

Figure 1. Effect on glucose tolerance of the diabetic rat by oral gavage of Shaddock fruit juice (SFJ) for 8 weeks

Table 3. Effect of SFJ treatment on lipid parameters of control and diabetic rats

\begin{tabular}{|c|c|c|c|c|c|c|}
\hline $\begin{array}{l}\text { Parameters, } \\
\mathrm{mg} / \mathrm{dL}\end{array}$ & $\mathrm{CN}$ & $\mathrm{CSFJ}_{25}$ & $\mathrm{CSFJ}_{50}$ & DC & $\mathrm{DSFJ}_{25}$ & $\mathrm{DSFJ}_{50}$ \\
\hline $\begin{array}{l}\text { Serum } \\
\text { cholesterol }\end{array}$ & $87.65 \pm 2.21$ & $90.05 \pm 0.57$ & $88.05 \pm 2.50$ & $120.45 \pm 2.25^{*}$ & $103.22 \pm 0.05$ & \\
\hline $\begin{array}{l}\text { HDL- } \\
\text { Cholesterol }\end{array}$ & $48.50 \pm 2.40$ & $49.35 \pm 0.70$ & $48.20 \pm 0.95$ & $25.45 \pm 0.30^{*}$ & $25.96 \pm 0.90$ & \\
\hline $\begin{array}{l}\text { Serum } \\
\text { Triglyceride }\end{array}$ & $98.95 \pm 2.20$ & $99.80 \pm 2.10$ & $100.50 \pm 2.10$ & $178.40 \pm 2.60^{*}$ & $166.30 \pm 0.88$ & $128.95 \pm 2.80^{\#}$ \\
\hline Serum & $3.45+0.60$ & & & $40.10 \pm 0.80^{*}$ & & $30.10 \pm 0.66^{\#}$ \\
\hline Serum LDL & $23.89 \pm 1.11$ & $25.90 \pm 0.89$ & $25.75 \pm 0.85$ & $66.75 \pm 3.45^{*}$ & $50.20 \pm 1.20$ & $35.18 \pm 1.05^{\#}$ \\
\hline
\end{tabular}

$C N=$ Normal control; $C_{C S F} J_{25}=$ Normal treated with $25 \%$ SFJ; $C S F J_{50}=$ Normal treated with $50 \%$ SFJ; DC = Diabetic control; $D S F J_{25}=$ Diabetic treated with $25 \%$ SFJ; $D S F J_{50}=$ Diabetic treated with 50\% SFJ, Values are expressed as Mean + S.E.M, * - significantly different from control $(p<0.05)$, \# - significantly different from diabetic control $(p<0.05)$

From the results of this study, it was found that there was increase in food and water intake and significant weight loss in diabetic animals as compared to control animals. $50 \%$ SFJ treatment significantly reduced the elevated food and water intake of the diabetic rats. The indication of this is that $50 \%$ SFJ may improve characteristic symptoms of polyphasia and polydypsia of diabetes mellitus. 


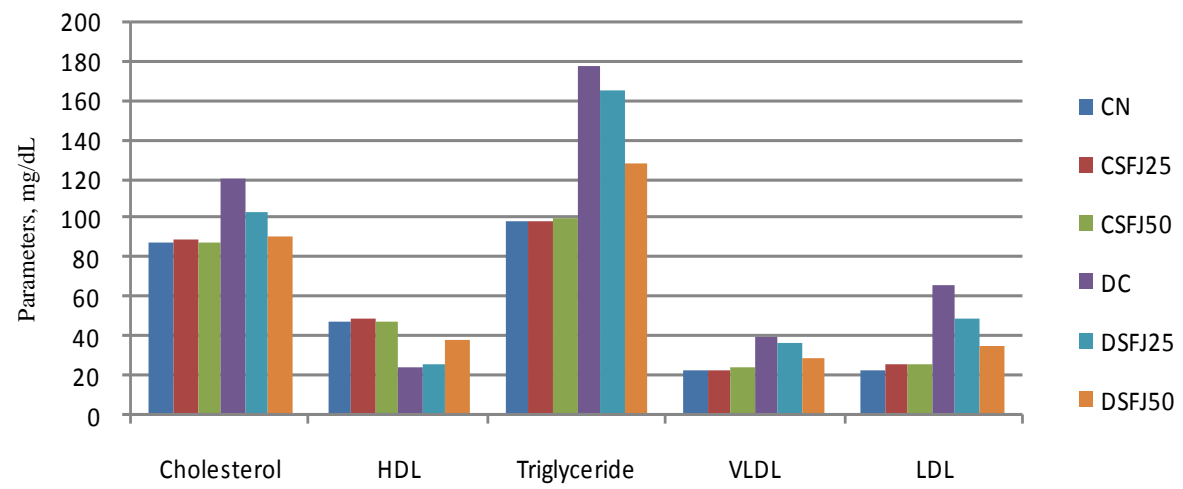

All values are means $\pm S . E(n=6) .{ }^{*} P<0.05$ compared with the native control group that reveals significant difference between two groups; \# $P<0.05$ compared with the diabetic group that reveals significant difference between two groups

Figure 2. Effects on lipid profile of the diabetic rat by oral gavage of Shaddock fruit juice (SFJ) for 8 weeks

Rosselin et al. $^{13}$ has reported that diabetic n2 STZ rats show significant glucose intolerance when orally administered with $1.5 \mathrm{~g} / \mathrm{kg}$ of glucose and also have high insulin level. This was confirmed by the present study as diabetic animals were found to have impaired glucosetolerance with high glucose level after one hour of glucose load compared to control animals. The results of this study have demonstrated that oral administration of $50 \%$ SFJ daily over duration of 8weeks to STZ-induced type-II diabetic rats can significantly improve the impaired glucose tolerance compare to $25 \%$ SFJ. These results are consistent with those reported earlier ${ }^{14,15}$.

Currently, the cellular mechanisms involved in the hypoglycaemic effects of Citrus maxima are not yet fully established. It is possible that SFJ can stimulate peripheral glucose utilization or it may inhibit key gluconeogenic enzymes such as glucose-6-phosphatase and fructose biphosphatase ${ }^{5}$.

Previous studies indicate that diabetes leads toalterations in the plasma lipid and lipoprotein profile and increases risk of coronary heart disease ${ }^{16,17}$. According to Brown et al. ${ }^{18}$, reducing the serum lipid levels through dietary or drug therapy decrease in the risk of cardiovascular disease and related complications.This study has demonstrated that SFJ possesses lipid lowering properties in diabetic animals in addition to the hypoglycemic activity. In the present study, serum cholesterol and triglyceride levels of diabetic rats were found to be significantly decreased by the treatment with $50 \%$ SFJ. It was also observed that in STZ induced diabetic rats; the level of HDL-cholesterol was significantly lower. These effects were normalized by $50 \%$ SFJ possibly by controlling the hydrolysis of certain lipoproteins and their selective uptake and metabolism by different tissues. The strong anti-hyperlipidemic effect of SFJ could also be through its control of hyperglycemia as this is a major determinant of total and very low density lipoprotein (LDL) and triglyceride concentration ${ }^{19}$. The lowering of cholesterol and triglyceride levels with increase in the HDL-cholesterol by 50\% SFJ may produce beneficial effects on STZ induced cardiovascular complications.

\section{Conclusion}

The results of this study suggest that SFJ prevents the induced metabolic abnormalities that 
Chem Sci Trans., 2013, 2(1), 19-24

normally accompany diabetes mellitus as evident from the reduction in cholesterol and triglyceride. These results have therefore demonstrated that Shaddock fruit juice can have marked beneficial effects in the treatment of diabetes mellitus. Shaddock fruit juice administration may therefore be useful as an adjunct therapy with oral hypoglycaemic agents in the management of diabetes mellitus.

\section{References}

1. American Diabetes Association, Diabetes Care, 2007, 30(1), S42-S47.

2. Kavishankar G B, Lakshmidevi N, Mahadeva- Murthy S, Prakash H S and Niranjana S R, Int J Pharm Biomed Sci., 2011, 2(3), 65-80.

3. Sinha A, Formica C, Tsalamandris C, Panagiotopoulos S, Hendrich E, DeLuise M, Seeman E and Jerums G, Diabetes Med.,1996, 13, 40-46.

4. Lucy D, Anoja S, Attele D D S and Chun-Su Y, Altern Med Rev., 2002, 7(1), 45-58.

5. Hongxiang H, George T and Vay LW Go, VLW, Chin Med., 2009, 4, 11-14.

6. Andrade-Cetto A. EstudioEtnobotánico y Fitoquímico de plantasútiles en la región de Xochipala Guerrero para el tratamiento de la diabetes NID, M.S. Thesis, Facultad de Ciencias, UNAM, Mexico, 1995.

7. Huang Y S and Ho S C, Food Chem., 2010, 119(3), 868-873.

8. Baker R A, Food Technol., 1994, 48(11), 133-138.

9. Sriparna K, Pallab KH, Malaya G, Upal KM, Prerona S, Asis B, Sanjib B and Biswakanth K, Endocrinology, 2011, DOI:10.5402/2011/869273.

10. Oyedepo T A, Sci J Med Clin Trials., 2012, DOI: 10.7237/sjmct/125.

11. Sitaswad S L, Shewad Y and Bhonde R, J Ethno Pharmacol., 2000, 73(1-2), 71- 79.

12. Friedewald W T, Levy R I and Fredrickson D S, Clin Chem., 1972, 18(6), 499-502.

13. Portha B, Picon L and Rosselin G, Diabetologia , 1979, 17, 371-377.

14. Sarkar S, Pranava M and Manita R, Pharmacological Research, 1996, 33, 1-4.

15. Ahmed I, Cummings E, Adeghate E, Sharma A K and Singh J, Mol Cell Biochem., 2004, 261(1-2), 63-70.

16. Taskinen M, Diabetes, 1992, 41, 12-17.

17. Betteridge J, Lipid disorders in diabetes mellitus, in: J.C. Pickup, G. Williams (Eds.), Text Book of Diabetes, $2^{\text {nd }}$ Ed., Blackwell Science, London, 1997, 55.1-55.31.

18. Brown G B, Xue-Qiao Z, Sacco D E and Alberts J J, Circulation, 1993, 87, 1781-1791.

19. Laakso M, Diabetic Rev., 1995, 3, 408-422. 